\title{
Reduction of Noise Spikes in Touch Screen Systems by Low Pass Spatial Filtering
}

\author{
Shuo Gao, Student Member, IEEE, David McLean, Jackson Lai, Charles Micou, and Arokia Nathan, Fellow, IEEE
}

\begin{abstract}
This paper reports on a low-pass spatial filtering technique for reduction of noise spikes in capacitive touch screen panels. Filter bandwidth is adjusted by dynamically evaluating attenuation of signal and noise spikes. Based on the experimental results, we boost the signal-to-noise ratio by $15.6 \mathrm{~dB}$ and attenuate noise spikes by $19.25 \mathrm{~dB}$. The processed signal yields higher detection accuracy and lower power consumption.
\end{abstract}

Index Terms-Capacitance touch screen panels (TSPs), low-pass spatial filtering, noise spike reduction and signal attenuation.

\section{INTRODUCTION}

$\mathbf{T}$ OUCH based human-machine interactivity has become a ubiquitous technology used in displays [1], [2]. Among different sensing techniques, capacitive touch screen panels (TSPs) are widely used in mobile devices, where high touch accuracy is a fundamental requirement for good user experience. Besides, the power consumption is another key parameter for long battery life time. A factor directly associated with these two parameters is the noise, which adversely affects the detection accuracy hereby increasing power consumption to reach/maintain a desired performance. Hence, the noise reduction is one of the main considerations in TSP design. Many noise reduction techniques are presented [3]-[10]. In this context, noise refers to any unwanted signals that may lead to detection errors. Noise conventionally includes deterministic (e.g., display noise) and stochastic (e.g., thermal noise) components. Traditionally offset on a single electrode is not considered as this can be cancelled by deducting a dc value. However, in capacitance TSPs, the global multi-valued offset gives rise to potential errors [11], which ought to be accounted for as a noise component. In our previous work [11], the global multi-valued offset is removed along with the common-mode noise by correlated double sampling (CDS) [12]-[14]. However,when the correlation between the touch frames and the noise reference frame becomes weak, noise spikes still remain, giving rise to detection errors. By analyzing the characteristics of the touch signal and noise spikes, we learn that the touch signal is normally of a low spatial frequency compared to the surrounding noise spikes [15]-[35]. Therefore spatial low-pass spatial filters can be used for the reduction of

Manuscript received November 09, 2015; revised January 07, 2016; accepted April 01, 2016. Date of publication April 05, 2016; date of current version August 15, 2016.

S. Gao, D. McLean, C. Micou, and A. Nathan are with the HeteroGenesys Laboratory, Department of Engineering, University of Cambridge, Cambridge CB3 0FA, U.K. (e-mail: sg690@cam.ac.uk; drm57@cam.ac.uk; cm704@cam.ac.uk; an299@cam.ac.uk).

J. Lai is with Research and Development Department, Ecmictics Biotech, Vaughan, ON L4L 4V8, Canada (e-mail: jlai@ecmictics.ca).

Color versions of one or more of the figures are available online at http://ieeexplore.ieee.org.

Digital Object Identifier 10.1109/JDT.2016.2550519 noise spikes. As depicted in Fig. 1, the noise spike is averaged by adjacent pixel values. However, the touch signal is reduced by the low pass spatial filtering as well, called smoothing effect, which may decrease signal-to-noise ratio (SNR), depending on the bandwidth and the mask size of the spatial filter. The induced smoothing effect may also result in signal distortion in terms of touch presence and position, leading to detection errors, thus requiring further research.

In this paper, we investigate the induced smoothing effect on touch detection. In particular, we analyze the following aspects: SNR, signal distortion (in terms of the change of touch position and the attenuation) and noise spike attenuation. The optimization of the above parameters can be achieved by tuning the mask size and bandwidth of the spatial filter. Based on the analysis, we present an adaptive bandwidth tuning algorithm for the dynamic optimization of spatial filter when the signal and noise conditions are changed. In the algorithm, we evaluate the attenuation of the signal and noise spike, and select a suitable bandwidth to maintain the desired performance.

This paper is structured as follows. Section II covers a review of the main noise sources and low pass spatial filters for TSPs. A theoretical analysis of the relationship between the mask size and registered touch position is provided in Section III. Section IV summarizes the parameters of the test bed and describes the low pass spatial filtering based adaptive bandwidth tuning algorithm. Section V contains experimental/simulation results and discussions. Finally, conclusionsare presented in Section VI.

\section{The MaIn Noise Sources In CAPACITIVE TSPS AND SPATIAL LOW PASS FILTERS}

\section{A. Charger Noise}

Ideally, the output from a chargerwould beconstant. However, in reality, the noise produced gives rise to common-mode fluctuation of the output, as shown in Fig. 2. An outstanding charger design can restrict common-mode noise below $3 \mathrm{~V}$, whereas a poorly designed charger can sometimes introduce peak common-mode noise higher than 40V [36]. ATSP can work smoothlywith common-mode noise when it is not being touched, as the difference of the charger output is maintained. There are two scenarios for touch events: the machine may be held by a hand, or placed on a table. In the former case, the machine may be sufficiently grounded to the earth thus no crucial common-mode noise charge escapes to the earth [36]. However, if the TSP is placed on a table, the touch panel and human body don't share the same ground, leading to potential touch mis-registrations. 


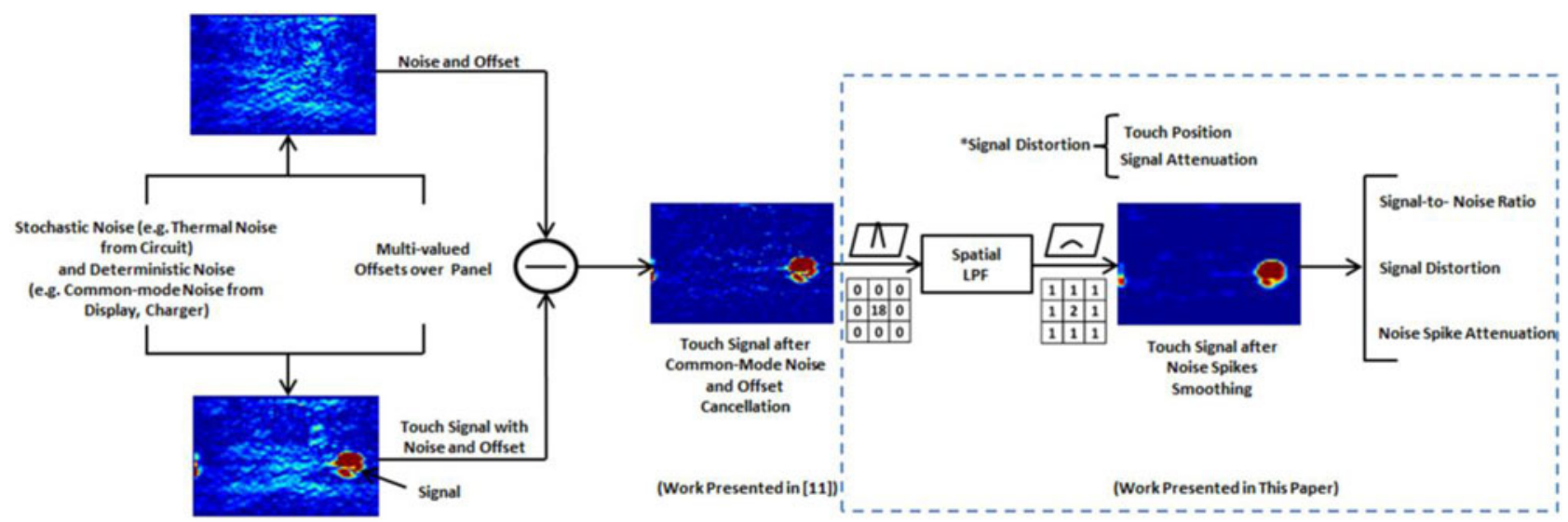

Fig. 1. Noise in touch screen systems; the CDS based technique for common-mode noise and global multi-valued offset cancellation; noise spikes attenuation by a spatial LPF and the corresponding smoothing effect.

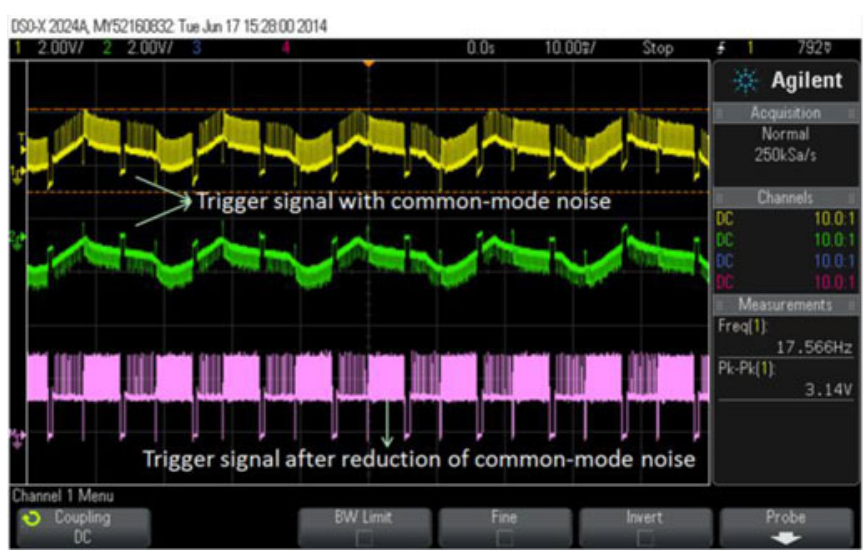

Fig. 2. The yellow signal (top) and the green signal (middle) are the trigger signals with common-mode noise introduced from the charger. The pink signal (bottom) is the trigger signal after common-mode noise reduction.

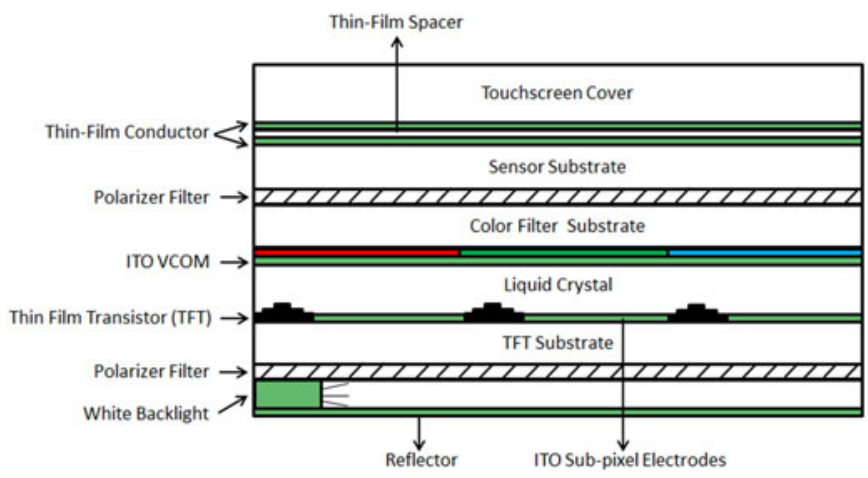

Fig. 3. Typical stack-up of a LCD pixel, modified from [37].

\section{B. Display Panel Noise}

Mainstream displays in today's portable digital assistants (PDAs) are active-matrix organic light-emitting diode [37], [38] and liquid crystal [39] architectures. The former one possesses excellent viewing quality and less noise for touch screen sensors. However it requires higher cost, hence, the popularity of LCDs is maintained. Fig. 3 depicts a typical stack-up of an LCD, showing that two capacitors are formed. The first is constituted

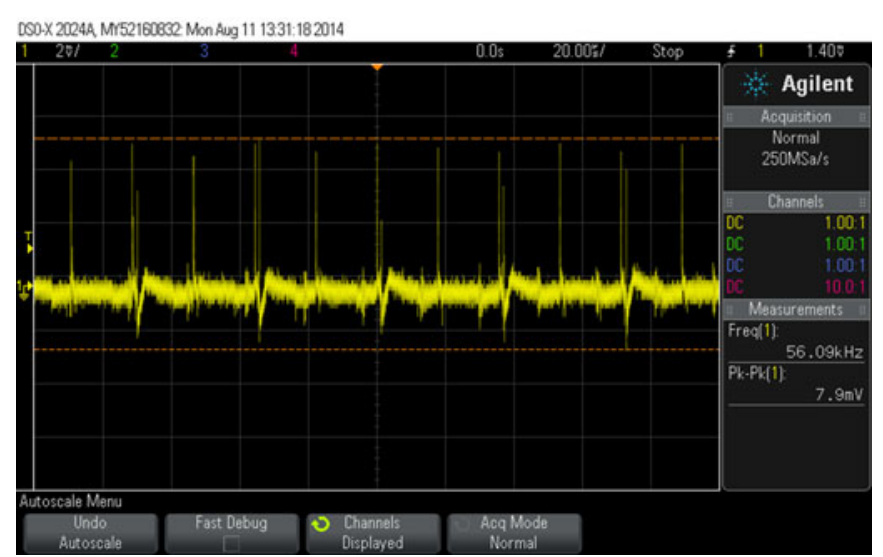

Fig. 4. LCD noise from the Dell e198wfp monitor (copper strip size was $3 \mathrm{~mm} \times 3 \mathrm{~mm})$.

of the sub-pixel electrode and the $\mathrm{V}_{\mathrm{COM}}$ layer, and shields the display noise since the $\mathrm{V}_{\mathrm{COM}}$ layer (e.g., made from ITO) has considerable resistance. The second one is constituted of the $\mathrm{V}_{\mathrm{COM}}$ layer and the sensing electrode, and couples the LCD noise to touch sensors. The coupled noise can be measured by detecting the voltage in a copper strip stacked directly on the LCD screen. The LCD noise from a commercial screen (Dell e198wfp) was measured using an oscilloscope (Agilent DSO-X 2024A). The area of the copper strip was $3 \mathrm{~mm} \times 3 \mathrm{~mm}$, which is in the same vein as that of a sensing electrode. Fig. 4 shows that the LCD noise involves a strong deterministic component, which mainly depends on the design of the product and does not significantly change after the product is fabricated [36]. Several methods are widely used to attenuate display noise: shielding, use of a different sensing frequency, and analog/digital filters.

\section{Low-Pass Spatial Filters and Corresponding Effect on Noise Reduction}

Spatial LPFs are divided into two categories: linear (e.g., average filter) and non-linear types (e.g., median filter) [40]-[44]. A mask/kernel with size $m \times n$ ( $m$ and $n$ are normally odd 

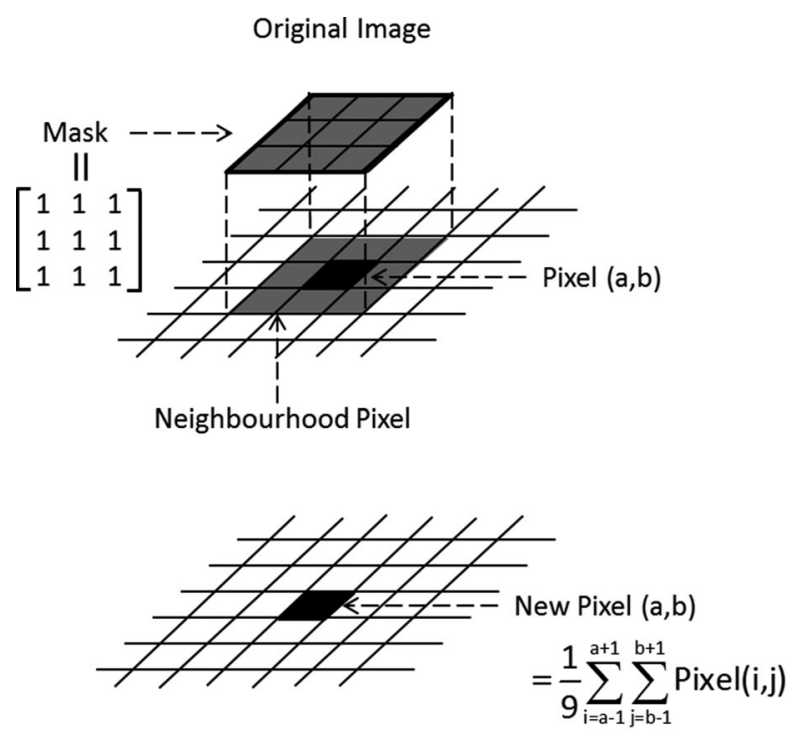

New Image

Fig. 5. Algorithm description of a spatially based average low-pass filter.

positive integers to ensure that there is only one pixel in the center of the mask) is employed in the spatial filter, using the same or different coefficients to control the bandwidth. Each pixel in the original image is computed along with its neighboring pixels, and the outcome is produced in a new image at the same position.

Both linear and non-linear structures offer benefits and drawbacks to noise spikes reduction for different types of touch based interactivities (e.g., finger touch). For example, good noise spike attenuation is offered by non-linear LPFs, which unfortunately may remove a stylus touch completely and cannot effectively remove the noise on the electrode. Thus, linear filters (average and Gaussian filter) are employed and analyzed in this paper. An example of an average filter is described in Fig. 5, whose mask size is $3 \times 3$ and the coefficients equal to 1 . Each pixel is added to the surrounding 8 pixels. The sum is then divided by the scaling constant 9 to generate a new pixel value.

In practice, this algorithm can be implemented by constructing an intermediate image in which each pixel contains the sum of 3 pixels in the $x$-direction. The final filtered image is obtained by performing the same process in the $y$-direction on the intermediate image, and then dividing by the scaling constant 9 . This process is equally valid for Gaussian-weighted filters. This is especially useful for higher resolution devices, which would require larger masks than $3 \times 3$, as this implementation scales linearly with mask size rather than quadratically.

The presented algorithm in this paper focuses on smoothing noise spikes to boost SNR while maintaining a desired signal strength level in order to avoid detection errors.

\section{THEORETICAL ANALYSIS}

\section{A. Mask Size and Touch Position}

To avoid the mis-registration at a wrong position and the interference from other touches, the mask size of the average filter is determined to be $3 \times 3$. This is because the touch event

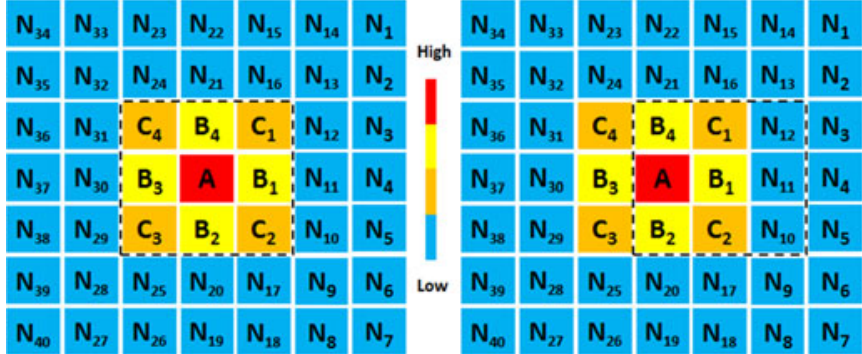

Fig. 6. Conceptual top-view of a finger touch on a mutual row-and-column capacitance TSP. $A, B_{1}$ to $B_{4}, C_{1}$, to $C_{4}$ and $N_{1}$ to $N_{4} 0$ indicate the values over the electrodes intersections. The average spatial filter is applied to the touch position pixel $\mathrm{A}$ (left) and the adjacent pixel $\mathrm{B}_{1}$ (right).

is limited in a certain region (e.g., $3 \times 3$ electrodes for a finger touch). If a largethe mask size is used, noise in the non-touch area weakens the strength of the touch signal, resulting in a high probability of touch mis-registration. Below we mathematically analyze the relationship between the mask sizes and touch position registration. Fig. 6 shows that a perpendicular finger touch occurring in the red region only affects the adjacent 8 pixels (yellow and green regions). A represents the value at the touch point, $B_{1}$ to $B_{4}$ and $C_{1}$ to $C_{4}$ are the adjacent region values, and $\mathrm{N}_{1}$ to $\mathrm{N}_{40}$ indicate the surrounding noise values. When a $3 \times 3$ average filter is applied, the output at the same position in the new image is

$$
A^{\prime}=\left(A+B_{1}+B_{2}+B_{3}+B_{4}+C_{1}+C_{2}+C_{3}+C_{4}\right) / 9 .
$$

If we assume that pixels at equal distance from the touch point have similar values, then (1) is simplified as

$$
A^{\prime}=\left(A+4 B_{1}+4 C_{1}\right) / 9 .
$$

In (2), $B_{1}$ and $C_{1}$ can be represented by $A$ with scaling down factors ( $b$ and $c$ ), expressed as (3)

$$
B_{1}=A+/ b, C_{1}=A+/ c,(b>1, c>1
$$

thus $A^{\prime}$ is expressed as (4)

$$
A^{\prime}=(A+4 A / b+4 A / c) / 9=A(b c+4 b+4 c) / 9 b c .
$$

If the registered touch position in the new image is shifted, this most likely happens within the yellow regions which are geographically closest to the red. The yellow region with value $B_{1}$ is analyzed to explain when the mis-registration in terms of position takes place. The filtered value $B_{1}^{\prime}$ is expressed as (5)

$$
\begin{aligned}
B_{1}{ }^{\prime} & =\left(A+B_{1}+B_{2}+B_{4}+C_{1}+C_{2}+N_{10}+N_{11}+N_{12}\right) / 9 \\
& \approx\left(A+3 B_{1}+2 C_{1}+3 N_{11}\right) / 9 \\
& =\left(A(b c+3 c+2 b)+3 b c N_{11}\right) / 9 b c .
\end{aligned}
$$

To ensure that $A^{\prime}>B_{1}^{\prime}$, the following condition must be satisfied:

$$
\frac{A}{N_{11}}>\frac{3 b c}{2 b+c}
$$


TABLE I

PARAmeters of Test Bed

\begin{tabular}{lcc}
\hline \hline Parameter & Unit & Value \\
\hline Diagonal & Inch & 10.1 \\
Aspect Ratio & None & $16 / 9$ \\
Excitation Voltage & Volt & 10 \\
Display Pixel Size & (Micrometer) $^{2}$ & $56 \times 56$ \\
TX Electrode Size & Millimeter & 3 \\
RX Electrode Size & Micrometer & 449 \\
Refreshing rate & Hertz & 60 \\
Sensing Array Size & (Millimeter $^{2}$ & $3 \times 3$ \\
Sensing Array Spacing & Millimeter & 2 \\
\hline \hline
\end{tabular}

*Note: TX and RX represent transmitter and receiver respectively.

Normally $N_{11}$ is about two orders smaller than $A$; and $b$ and $c$ lie between 1 to 3. Hence, the condition expressed in Eq. 6 is satisfied. When the mask size increases to $5 \times 5$, the condition of $A^{\prime}>B_{1}^{\prime}$ is completely determined by the surrounding noise values, resulting in a high probability of mis-registration.

\section{B. Spatial Frequency Properties of Signal and Noise Spikes}

Touch signal is normally low spatial frequency compared to the noise spikes. This is the fundamental assumption of low pass spatial filtering technique. However, in some cases the touch signal can offer high spatial frequency as well. For example, only one electrode may be affected when a stylus touch is applied. In contrast, when a noise spike happens within the touch region, it may have low spatial frequency property. The low pass spatial filtering cannot remove noise spikes and may result in the decrement of SNR when the spatial frequencies of touch signal and noise spikes are very close or overlapped. A possible solution of this is the time domain low pass filtering at pixel level.

In this paper, finger touch is used and analyzed, as this is the most preferred touch activity for PDAs. Finger touch is of low spatial frequency, and the bandwidth relies on the contact area, which is highly individual dependent. Even for the same user, the touch property can be changed when different applications are used. Hence, the filter bandwidth should be dynamically adjusted to keep the desired touch information while maximally reducing noise spikes.

\section{EXPerimental Test Bed ANd Algorithm Description}

The experiments were carried out on an $80 \times 80 \mathrm{TSP}$, and the details are provided in Table I.

To reduce the noise spikes in the frame after CDS [11], a spatial LPF with an initial bandwidth is used. Since the touch and noise properties are dynamically changed, the bandwidth of the spatial LPF is required to be adjusted in order to optimize the performance. As described in Fig. 7, the frame after CDS (denoted as $f_{\mathrm{CDS}}$ ) is send to the spatial LPF for the noise spikes reduction. Then the filtered frame (denoted as $f_{\mathrm{LPF}}$ ) enters into the touch decision function to determine if a touch happens or not. If a touch is registered, then the frame (denoted as $f_{\text {Touch }}$ ) is evaluated in terms of the signal and noise spike attenuation by the bandwidth decision function, to analyze if the bandwidth of the spatial LPF needs to be changed. If no touch is regis-

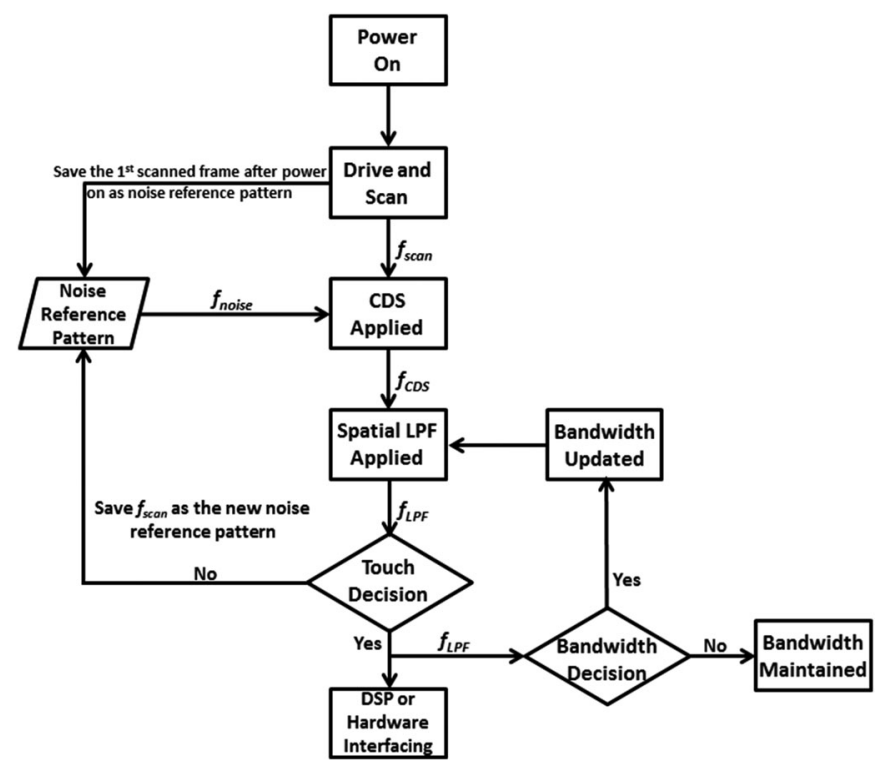

Fig. 7. Flowchart of the CDS and spatial LPF based noise reduction algorithm.

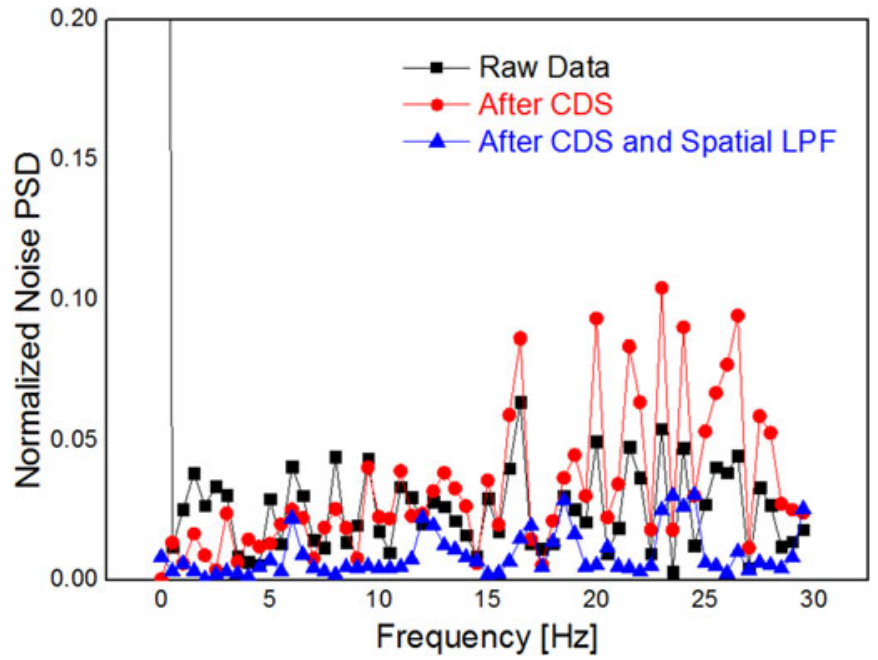

Fig. 8. Normalized PSD plots of the original output, CDS output with sampling frequency at $60 \mathrm{~Hz}$ and spatial average LPF output with mask size of $3 \times 3$.

tered, the scanned frame $\left(f_{\text {scan }}\right)$ is updated as a new reference noise pattern, which will be used by CDS algorithm, as shown in Fig. 7.

\section{RESULTS AND DISCUSSION}

To evaluate the smoothing effect, three factors are considered here: SNR, signal and noise attenuation. The normalized output (no touch event) PSD plots (see Fig. 8) are used to analyze the noise behavior after the average LPF. It can be observed that the high frequency noise generated by the CDS is suppressed. The SNR is boosted by $15.6 \mathrm{~dB}$. The signal and noise spike are attenuated by 4.51 and $19.25 \mathrm{~dB}$ respectively. The attenuation of the signal strength is undesirable, thus different coefficients are used for the bandwidth adjustment to retain more signal 


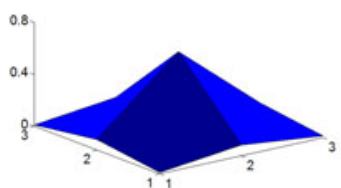

(a)

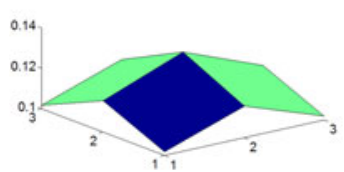

(c)

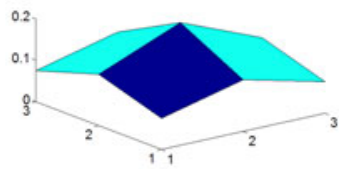

(b)

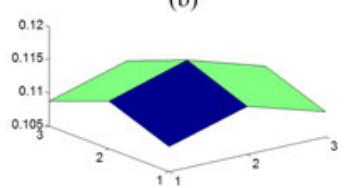

(d)
Fig. 9. Four Gaussian distribution based masks with different bandwidths (represented by standard deviations). (a) $\sigma=0.5$; (b) $\sigma=1$; (c) $\sigma=2$; (d) $\sigma=4$.

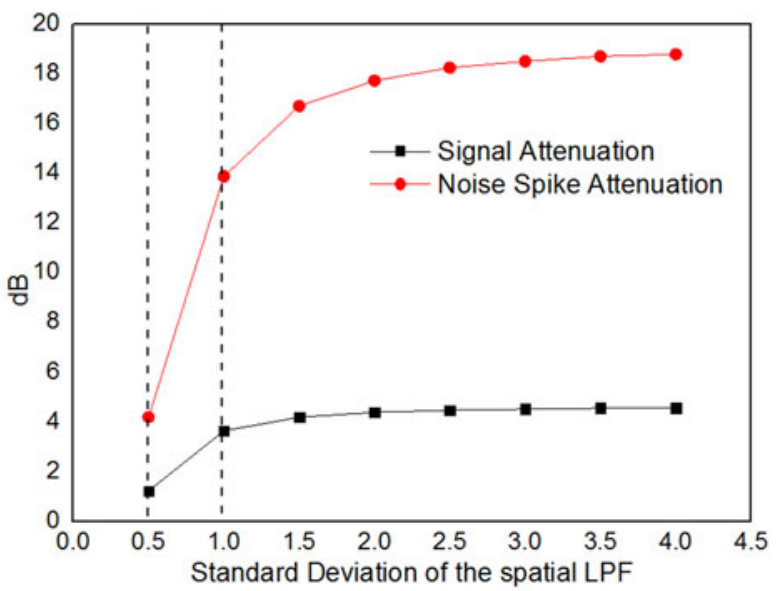

(a)

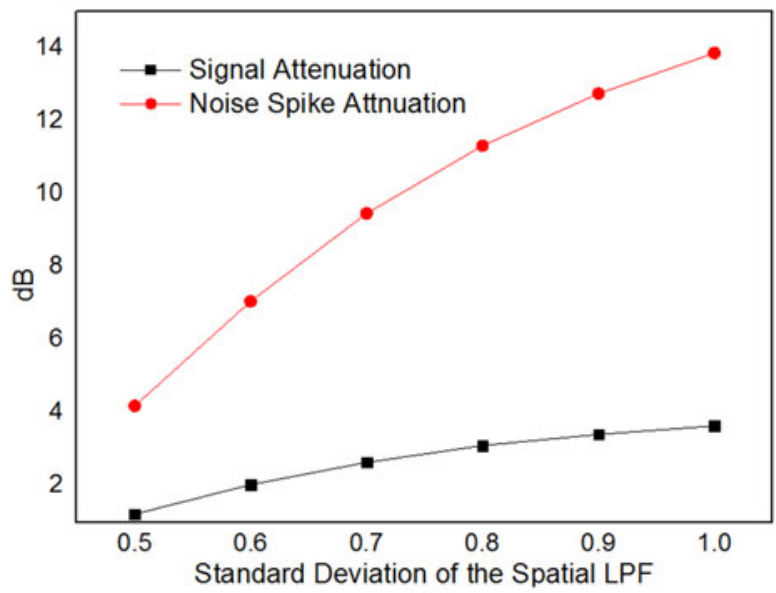

(b)

Fig. 10. Results of signal and noise spike attenuation by applying spatial LPFs with different standard deviations. (b) is the dashed part of (a).

information. The coefficients of the filter mask follow Gaussian distribution with different standard deviations $(\sigma)$, representing various bandwidths of the LPF. Four Gaussian distribution based filter masks are illustrated in Fig. 9. Here small standard deviation indicates that more information about the pixel itself is maintained. In contrast, large standard deviation implies that the pixel is strongly affected by the adjacent neighborhoods. The simulation results of applying spatial filter with different bandwidths are illustrated in Fig. 10. Due to the spatial high frequency property, noise spike suffers severer attenuation compared to the touch signal. From Fig. 10(a), the signal and noise spike are attenuated dramatically when $\sigma$ is within the range of 0.5 to 1 . After $\sigma>1.5$, the trends of attenuation are reaching saturation. Thus the range of $0.5<\sigma<1$ is further investigated, and the results are illustrated in Fig. 10(b). By evaluating the signal and noise spike attenuation dynamically, the filter bandwidth is updated. For example, if the accepted signal attenuation is $2 \mathrm{~dB}$, then the initial Gaussian distribution based mask with standard deviation at 0.6 would be used to maximally attenuate the noise spike. Later, when the signal attenuation is found to be $1 \mathrm{~dB}$, then a bigger standard deviation can be selected to further smooth noise spikes and boost SNR.

It is also important to investigate the computational time and energy consumption of the presented algorithm. Our system's scanning rate is $60 \mathrm{~Hz}$, thus the computational time of the algorithm should be much lower than $16.7 \mathrm{~ms}$. The required computational time depends on the complexity of the algorithm and the performance of the processor. The complexity of the spatially based algorithms used in this research is around $O(3 N)$, where $N$ is equal to 6400 . Current commercial processors in mobile phones can operate in the range of GHz. Hence, a computational time of approximately $19.2 \mu \mathrm{s}$ is required when a $1 \mathrm{GHz}$ processor is equipped. Commercial processors can work at $20 \mathrm{MIPS} / \mathrm{mW}$ [45], therefore the power consumption of the algorithm is $21.6 \mathrm{nW}$ which is negligible compared to that of scanning the whole panel.

\section{CONCLUSION}

The noise spikes in TSPs give rise to "fake" touch, resulting in the high power consumption. In this paper, we present a low pass spatial filtering based technique for the noise spike reduction. By the approach of evaluating the spatial LPF induced smoothing effect, the filter bandwidth is dynamically adjusted to optimize the performance. Based on the experiment, a SNR enhancement of $15.6 \mathrm{~dB}$ and a noise spike attenuation of $19.25 \mathrm{~dB}$ are obtained. The filtered signal improves detection accuracy, thus less energy is required to maintain a desired performance.

\section{REFERENCES}

[1] L. Zhang, J. Saboune, and A. E. Saddik, "Transforming a regular screen into a touch screen using a single webcam," J. Display Technol., vol. 10, no. 8, pp. 55-8, Aug. 2014.

[2] A. Nathan, J. C. S. Lai, S. Cha, and C. Church, "Pressure sensing display device," U.S. Patent US20140008203 A1, Jan. 9, 2014.

[3] K. Kim et al., "A capacitive touch controller robust to display noise for ultrathin touch screen displays," in Proc. IEEE Int. Solid-State Circuits Conf. Dig Tech. Papers, Feb. 2012, pp. 116-117.

[4] J. E. Park, D. H. Lin, and D. K. Jeong, "A reconfigurable 40-to-67 dB, 50to-6400 Hz frame-rate, column-parallel readout IC for capacitive touchscreen panels," IEEE J. Solid-State Circuits, vol. 49, no. 10, pp. 23052318, Oct. 2014.

[5] S. Ko, H. Shin, H. Jang, L. Yun, and K. Lee, “A 70 dB SNR capacitive touch screen panel readout IC using capacitor-less trans-impedance amplifier and coded orthogonal frequency-division multiple sensing scheme," in Proc. IEEE Symp. Very Large Scale Integr., 2013, pp. C216-C217.

[6] S. Ko et al., "Low noise capacitive sensor for multi-touch mobile handset's applications," in Proc. IEEE Asian Solid State Circuits Conf., Nov. 2010, pp. $1-4$.

[7] H. Jang, H. Shin, S. Ko, I. Yun, and K. Lee, "2D coded-aperture-based ultra-compact capacitive touch-screen controller with 40 reconfigurable 
channels," in Proc. IEEE Int. Solid-State Circuits Conf. Dig. Tech. Papers, Feb. 2014, pp. 218-219.

[8] J. Yang et al., "A highly noise-immune touch controller using FilteredDelta-Integration and a charge-interpolation technique for 10.1-inch capacitive touch-screen panels," in Proc. IEEE Int. Solid-State Circuits Conf. Dig. Tech. Papers, Feb. 2013, pp. 390-391.

[9] N. Miura et al., "A $1 \mathrm{~mm}$-pitch $80 \times 80$-channel $322 \mathrm{~Hz}$-frame-rate touch sensor with two-step dual-mode capacitance scan," in Proc. IEEE Int. Solid-State Circuits Conf. Dig. Tech. Papers, 2014, pp. 216-217.

[10] H. Shin, S. Ko, H. Jang, I. Yun, and K. Lee, "A 55dB SNR with $240 \mathrm{~Hz}$ frame scan rate mutual capacitor $30 \times 24$ touch-screen panel read-out IC using code-division multiple sensing technique," in Proc. IEEE Int. Solid-State Circuits Conf. ISSCC Dig. Tech. Papers, 2013, pp. 388-389.

[11] S. Gao, J. Lai, C. Micou, and A. Nathan, "Reduction of common-mode noise and global offsets in touch screens by correlated double sampling," J. Display Technol.,vol.12, no. 6, Jun. 2016.

[12] M. H. White and D. R. Lampe, "Characterization of surface channel CCD image arrays at low light levels," IEEE J. Solid-State Circuits, vol. SC-9, no. 1, pp. 1-13, Feb. 1974.

[13] C. Enz and G. Temes, "Circuit Techniques for reducing the effects of opamp imperfections: Autozeroing, correlated double sampling, and chopper stabilization," Proc. IEEE, vol. 84, no. 11, pp. 1584-1614, Nov. 1996.

[14] J. Ohta, Smart CMOS Image Sensors and Applications. Boca Raton, FL, USA: CRC Press, 2007.

[15] W. C. Westerman and S. Hotelling, "Perpheral pixel noise reduction," U.S. Patent US7643010 B2, Jan. 5, 2010.

[16] S. Hotelling et al., "Gestures for touch sensitive input devices," U.S. Patent US20060026521 A1, Feb. 2, 2006.

[17] B. R. Land and S. P. Hotelling, "Error compensation for multi-touch surfaces," U.S. Patent US7692638 B2, Apr. 2010.

[18] Z. Baharav and R. Kakarala, "Capacitive touch sensing: Signal and image algorithms," Proc. SPIE, vol. 7873, pp. 78730H-1-78730H-12, Feb. 2011

[19] H. Akhtar and R. Kakarala, "A methodology for evaluating accuracy of capacitive touch sensing grid patterns," J. Display Technol., vol. 10, no. 8, pp. 672-682, Aug. 2014.

[20] T. Wang, Y. Ko, and C. Lai, "Gesture detecting method capable of filtering panel mistouch,” U.S. Patent US20130093692, Apr. 2013.

[21] B. O. Hagermoser et al., "Touch panel system and method for distinguishing multiple touch inputs," U.S. Patent US7254775 B2, Aug. 7, 2007.

[22] J. Jordan, "Sensing and defining an input object," U.S. Patent US20110057670 A1, Mar. 10, 2011.

[23] I. Guarneri, A. Capra, G. M. Farinella, and S. Battiato, "Shape recognition for capacitive touch display," SPIE Electron.Imag., Mar. 2013.

[24] W. C. Westerman, "Image jaggedness filter for determining whether to perform baseline calculations," U.S. Patent US20090174688 A1, Jul. 9 , 2009.

[25] W. C. Westerman, "Multi-touch input discrimination," U.S. Patent US20080158185 A1 Jul. 3, 2008

[26] I. Guarneri, C. Capra, A. Castorina, S. Battiato, and M. G. Farinella, "PCA based shape recognition for capacitive touch display," in Proc. IEEE Int. Conf. Consumer Electron., 2013, pp. 1-2.

[27] J. Yang et al., "A noise-immune high-speed readout circuit for in-cell touch screen panels," IEEE Trans. Circuits Syst. I, Reg. Papers, vol. 60, no. 7, pp. 1800-1809, Jul. 2013.

[28] S. P. Hotelling et al., "Ground detection for touch sensitive device," U.S. Patent WO2011008610 A1, Jan. 20, 2011.

[29] H. S. Lee, T. J. Park, and K. J. Kim, "Touch sensing method and touch sensing device," U.S. Patent US20140139478 A1, May 22, 2014.

[30] J. Zhang, Y. Guo, and L. Mo, "Multi-touch detection method for capacitive touch screens," U.S. Patent US20110221701 A1, Sep. 15, 2011.

[31] B. R. Land, M. Yousefpor, and S. P. Hotelling, "Negative pixel compensation," U.S. Patent US8570301 B2, Oct. 29, 2013.

[32] J. Liu, L. Liu, C. Chang, and S. Tai, "Touch device and driving method of touch panel thereof," U.S. Patent US20140145965 A1, May 29, 2014.

[33] H. Philipp, "Capacitive sensor and array," U.S. Patent US6452514 B1, Sep. 15,2002

[34] T. Hwang, W. Cui, I. Yang, and O. Kwon, "A highly area-efficient controller for capacitive touch screen panel systems," IEEE Trans. Consum. Electron., vol. 56, no. 2, pp. 1115-1122, May 2010.

[35] J. H. Yang, J. M. Choi, Y. S. Kim, and H. S. Oh, "Control circuit of touch screen and noise removing method," U.S. Patent US20130300690 A1, Nov. 14, 2013.

[36] H. W. Klein, "Noise immunity of touchscreen devices," White Paper, Cypress Semiconductor Corp., San Jose, CA, USA, Feb. 2013.
[37] C. M. Lai, Y. H. Yeh, and Y. H. Huang, "Pixel structure of active matrix organic light-emitting diode and method for fabricating the same," U.S. Patent US20070152217 A1, Jul. 5, 2007.

[38] E. Anderson, "Reduce display noise in capacitive touchscreens," White Paper, Cypress Semiconductor Corp., San Jose, CA, USA, Jan. 2013.

[39] S. Kokawa, M. Nishihara, and Y. Sato, "Liquid crystal display," U.S Patent US5467208 A, Nov. 14, 1995.

[40] A. McAndrew, "An introduction to digital image processing with MATLAB," Lecture note for SCM2511 Image Processing, Boston, MA, US: Course Technology Press, Univ. of Technology, Sep. 2004.

[41] J. R. Jensen and K. Lulla, "Introductory digital image processing: A remote sensing perspective," Geocarto Int., vol. 2, no. 1, pp. 65-65, 1987.

[42] J. C. Russ, "The image processing handbook," J. Comput. Assist. Tomogr., vol. 19, no. 6, pp. 979-981, 1995.

[43] J. S. Lim, Two-Dimensional Signal and Image Processing. Englewood Cliffs, NJ, USA: Prentice-Hall, 1990.

[44] J. S. Lee, "Digital image enhancement and noise filtering by use of local statistics," IEEE Trans. Pattern Anal. Mach. Intell., vol. PAMI-2, no. 2, pp. 165-168, Mar. 1980.

[45] ARM. Cortex-a5 processor performance. [Online]. Available: http:// www.arm.com/products/processors/cortex-a/cortex-a5.php, last retrieved on 23rd Apr 2016.

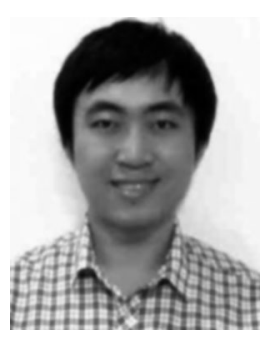

Shuo Gao received the M.Sc. degree in electrical engineering from the University of Ottawa, Ottawa, ON, Canada, 2013 and currently currently working toward the Ph.D. degree in the Hetero-Genesys Laboratory, University of Cambridge, Cambridge, U.K.

Previously, he was an Optical Fiber System Engineer at Ciena Corporation, Ottawa, ON, Canada. His research interests include touch interactivity and RF system for flexible electronics.

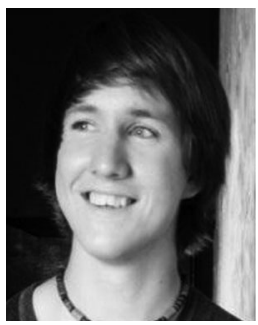

David McLean received the M.Eng. degree in engineering from the University of Cambridge, Cambridge, U.K., in 2014, specialising in information and data processing.

His areas of interest include image processing, computer vision, machine learning, and speech and language processing.He was a Technology Scholar at Cambridge Consultants Ltd and is a Member of the Institution of Engineering and Technology.

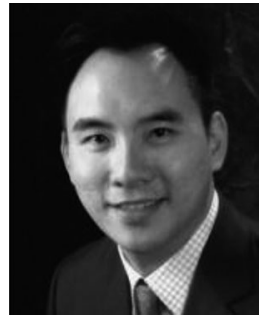

Jackson Lai received the Ph.D. degree in electrical engineering from the University of Waterloo, Waterloo, ON, Canada, in 2007.

$\mathrm{He}$ is currently with the Research and Development Department, Ecmictics Biotech, Vaughan, ON, Canada. previously, he was a Senior Display Technology Developer at Blackberry. he has held positions such as Device and Circuit Engineer in Carestream Health, as well as Lead Display Circuit Designer in Ignis Innovation Inc. His research interests include imaging circuits, large area electronics, as well as system on panel design. 


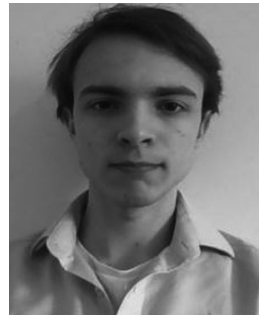

Charles Micou is working toward the M.Eng. degree from the University of Cambridge, U.K.,

After two years of general engineering, he specialized into electrical and information sciences. His areas of interest include computer vision, machine learning, image processing, and embedded systems. $\mathrm{He}$ has worked as a Software Engineer on projects ranging from embedded telecommunications devices at Eseye, to cloud-based distributed simulations at Improbable.

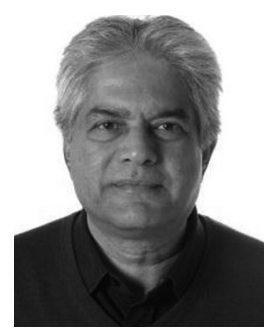

Arokia Nathan (S'84-M'87-SM'99-F'10) received the $\mathrm{Ph} . \mathrm{D}$. degree in electrical engineering from the University of Alberta, Edmonton, AB, Canada.

Following post-doctoral years at LSI Logic Corp., USA, and ETH Zurich, Switzerland, he joined the University of Waterloo, Canada, where he held the DALSA/NSERC Industrial Research Chair in sensor technology and subsequently the Canada Research Chair in nano-scale flexible circuits. In 2006, he moved to the U.K. to take up the Sumitomo Chair of Nanotechnology at the London Centre for Nanotechnology, University College London, where he received the Royal Society Wolfson Research Merit Award. He has held Visiting Professor appointments at the Physical Electronics Laboratory, ETH Zürich, and the Engineering Department, Cambridge University, U.K. He holds the Chair of Photonic Systems and Displays in the Department of Engineering, Cambridge University. He has published more than 500 papers in the field of sensor technology, CAD, thinfilm transistor electronics, and is a coauthor of four books. He has more than 50 patents filed/awarded and has founded/cofounded four spin-off companies. He serves on technical committees and editorial boards in various capacities.

Dr. Nathan received the 2001 NSERC E.W.R. Steacie Fellowship. He is a Chartered Engineer (U.K.), Fellow of the Institution of Engineering and Technology (U.K.), and an IEEE/EDS Distinguished Lecture. 